\title{
Parameter dependences of the separatrix density in nitrogen seeded ASDEX Upgrade H-mode discharges
}

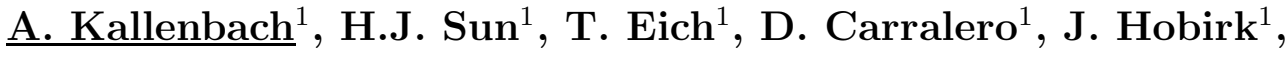 \\ A. Scarabosio ${ }^{1}$, M. Siccinio ${ }^{1}$, ASDEX Upgrade team ${ }^{2}$, \\ EUROfusion MST1 team ${ }^{3}$ \\ ${ }^{1}$ Max Planck Institute for Plasma Physics, D-85748 Garching, Germany, \\ ${ }^{2}$ A. Kallenbach et al., Nucl. Fusion 57 (2017) 102015 \\ ${ }^{3}$ H. Meyer et al., Nucl. Fusion 57 (2017) 102014
}

\begin{abstract}
The upstream separatrix electron density is an important interface parameter for core performance and divertor power exhaust. It has been measured in ASDEX Upgrade $\mathrm{H}$-mode discharges by means of Thomson scattering using a self-consistent estimate of the upstream electron temperature under the assumption of Spitzer-Härm electron conduction. Its dependence on various plasma parameters has been tested for different plasma conditions in H-mode. The leading parameter determining $\mathrm{n}_{e, s e p}$ was found to be the neutral divertor pressure, which can be considered as an engineering parameter since it is determined mainly by the gas puff rate and the pumping speed. The experimentally found parameter dependence of $\mathrm{n}_{e, s e p}$, which is dominated by the divertor neutral pressure, could be approximately reconciled by 2 -point modelling.
\end{abstract}




\section{Introduction}

A very important interface parameter between core plasma performance and divertor power exhaust is the electron density at the outer midplane separatrix, $\mathrm{n}_{e, s e p}$. In a most simple and qualitative picture, a low separatrix density is beneficial for the plasma energy confinement [1] [2], while a high density enables and supports the achievement of divertor detachment [3] [4] [5] [6]. An important tokamak operational limit, the H-mode density limit, is also connected to an upper limit of the separatrix density [7] [8]. The power exhaust is also closely coupled to the divertor neutral pressure or neutral fluxes: The power flux in the outer divertor at the detachment threshold was found to scale about linearly with the weighted pressures of deuterium and nitrogen [9] [10]. Neutral divertor fluxes can be regarded as superior parameters for the description of power dissipation since the divertor losses are closer linked to fluxes than to densities (relevance of the radiative potential [11] [12] instead of loss function $\mathrm{L}_{z}$ ). In addition, impurity fluxes are better known in the divertor in comparison to impurity concentrations. Therefore, it is of great interest to establish the relation between divertor neutral pressure and the upstream separatrix density in order to achieve a better integration of energy confinement and exhaust optimization. The use of the measured pressure in combination with simple models is also motivated by problems reported from SOLPS modelling which significantly underestimates the divertor neutral pressure while matching the plasma profiles for conditions similar to those of this paper [13].

Direct measurements of the (upstream) separatrix density are not easily available. The major experimental problem stems from the correct assignment of the separatrix position from equilibrium reconstruction, which suffers uncertainties comparable to the radial density decay length. To overcome this problem, Thomson scattering is used which measures $T_{e}$ and $n_{e}$ at the same location, while the separatrix position is assigned via the power flux $\mathrm{P}_{\text {sep }}$ and an assumption for the width of the power carrying layer [14] [15] [16] [17].

The lack of a large experimental data base of the separatrix density is the reason for a lack of scalings of this quantity with engineering parameters like machine radius $\mathrm{R}$ or plasma current $\mathrm{I}_{p}$. Based on experience on present day tokamaks, a constant fraction of separatrix density over Greenwald density, $\mathrm{n}_{e, s e p} / \mathrm{n}_{G W}$ is often assumed for detachment onset [5]. Due to the large $\mathrm{I}_{p}$ ratio between current experiments and, e.g., ITER, the parameter dependence of $\mathrm{n}_{e, s e p}$ on $\mathrm{I}_{p}$ is of particular importance. Regarding power exhaust, in addition to the separatrix density, the power width $\lambda_{q}$ and the divertor broadening $\mathrm{S}$ are important [10]. $\lambda_{q}$ was found to scale roughly with the inverse poloidal magnetic field, $1 / \mathrm{B}_{p}$, and thus $\lambda_{q} \propto 1 / \mathrm{I}_{p}$ for constant geometry [18]. Regarding the divertor broadening $\mathrm{S}$, no measurements are available for conditions with significant divertor losses or detached conditions.

In this paper, an experimental data base is presented of nitrogen seeded AUG Hmode discharges with sufficiently constant seeding level for the achievement of nearly 
steady state conditions and $\mathrm{n}_{e}, \mathrm{~T}_{e}$ measurements by the edge Thomson scattering system [19]. The experimental data base is described in section 2. Emphasis is placed on the divertor neutral pressure, which can be regarded as an engineering parameter in devices with a closed divertor and active pumping. Simple 2-point considerations are presented in section 3 for the relation of the upstream separatrix density and the divertor neutral flux. The spatial neutral flux distribution, which is effected by the high field side high density region (HFSHD) [20] is discussed in section 4. Some conclusions are drawn in section 5 .

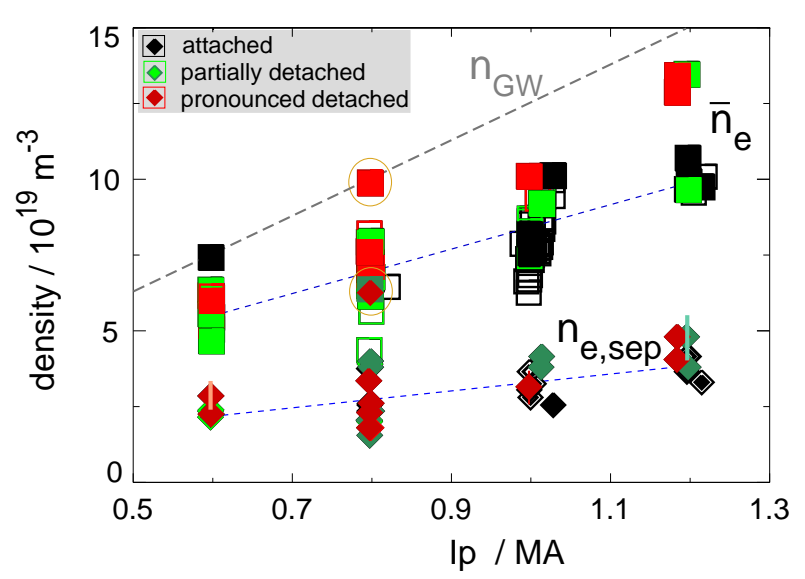

Figure 1. Line-averaged (squares) and separatrix (darker color, diamonds) densities versus plasma current in the data base. Full symbols denote data base entries with $n_{e, s e p}$ data. Blue dotted lines are (offset) linear fits to guide the eye. Golden circles denote line-averaged and separatrix density very close to the $H$-L density limit, \# 34613 $t=3.8-3.95 \mathrm{~s}$.

\section{Experimental data base}

When inspecting outer divertor detachment behaviour, the most important input is the parallel heat flux arriving at the divertor entrance. Its radial decay length, $\lambda_{q}$, has been very well characterized in the past using IR thermography, starting from the divertor surface side, and robust scalings for fully attached H-mode conditions are available [18] [21]. The target heat flux can for sufficiently collisional conditions be related to the power flux crossing the separatrix, $\mathrm{P}_{\text {sep }}$, assuming Spitzer-Härm parallel electron heat conduction [19]. For conditions of non-negligible divertor radiation or momentum losses, IR measurements can no longer be directly related to the heat flux at the divertor entrance. These are the conditions of interest in this study. A data base was set up of ASDEX Upgrade H-mode discharges from 2014-2017 (divertor III) with different deuterium gas puff and nitrogen seeding levels, plasma currents and separatrix crossing power, $\mathrm{P}_{\text {sep }}$. Time intervals of typically $0.5 \mathrm{~s}$ duration are considered, where $\mathrm{D}$ and $\mathrm{N}$ gas puff levels are sufficiently stationary and in balance with pumping. Of special interest here are discharges with good quality of the edge Thomson scattering data for the determination of electron temperature and density fall-off widths, $\lambda_{T e}$ and $\lambda_{n}[19]$. 
Thomson scattering data are evaluated using a simple 2-point scaling prescribing the upstream electron temperature. Exponential decays are fitted to the edge Thomson scattering $\mathrm{n}_{e}, \mathrm{~T}_{e}$ data around the separatrix position. The conducted power flux to the outer target is estimated by the difference of the absorbed heating power and the total radiated power. The conducted power flux to the inner divertor is assumed to be completely radiated. Taking $2 / 7 \lambda_{T e}$ as the measured power width, $\mathrm{T}_{e, s e p}$ is then derived assuming Spitzer-Härm parallel electron conductivity [17]. $\mathrm{n}_{e, s e p}$ is taken from the Thomson profile fit data at the location where $\mathrm{T}_{e, s e p}$ is measured. The range of $\mathrm{T}_{e, s e p}$ in the present database is from 73 to $148 \mathrm{eV}$.

Figure 1 shows the line-averaged and the separatrix densities of the data base versus the plasma current. With significant variations, both densities rise roughly proportional to the plasma current. The highest line-averaged densities approaching the Greenwald density are obtained under pronounced detachment conditions [9]. The uncertainty of $n_{e, s e p}$ is typically $+15,-20 \%$, where the asymmetry results from the assumption that the fraction of $\mathrm{P}_{\text {sep }}$ flowing to the outer divertor is delivered to the electron channel, thus $\mathrm{T}_{e, s e p}$ being at its upper limit and hence $\mathrm{n}_{e, s e p}$ evaluated at a high value, accordingly. Figure 2 compares the Spitzer-Härm power decay length with the prediction of the
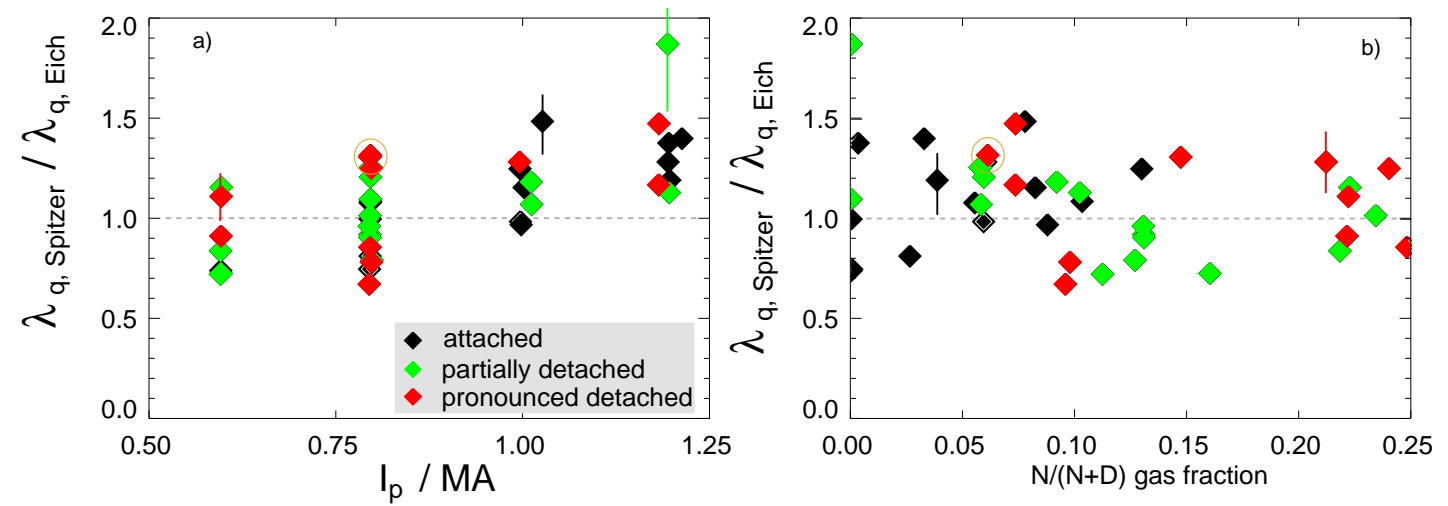

Figure 2. a) Comparison of power decay lengths $\lambda_{q}$ derived from Thomson scattering assuming Spitzer-Härm parallel electron heat conduction with the prediction of the Eich scaling for discharges with partially strong $N$ and $D$ puffing and different degrees of detachment. Absolute values of the reference $\lambda_{q, \text { Eich }}$ vary from about $3.3 \mathrm{~mm}(0.6$ $M A)$ to $1.7 \mathrm{~mm}$ (1.2 MA) measured in radial direction in the outer midplane. Error bars denote typical statistical errors, a possible contribution of ELMs to the derived

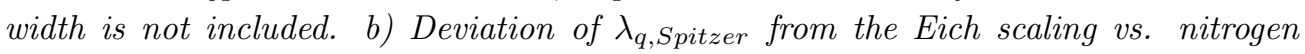
atomic fraction in the divertor gas. No trend with the gas fraction, which approximates the $N$ concentration in the divertor plasma [10], is observed. $P_{\text {heat }}=2 \ldots 25 \mathrm{MW}$, $P_{\text {sep }}=1.4 \ldots 20 \mathrm{MW}$.

Eich scaling [18] for nitrogen seeded discharges with different gas puff levels, heating powers, plasma current etc. Due to the partly very high ELM frequencies and small ELM amplitudes, no effort was taken to cut out ELM effected time points from the Thomson scattering data, as done in [19]. 


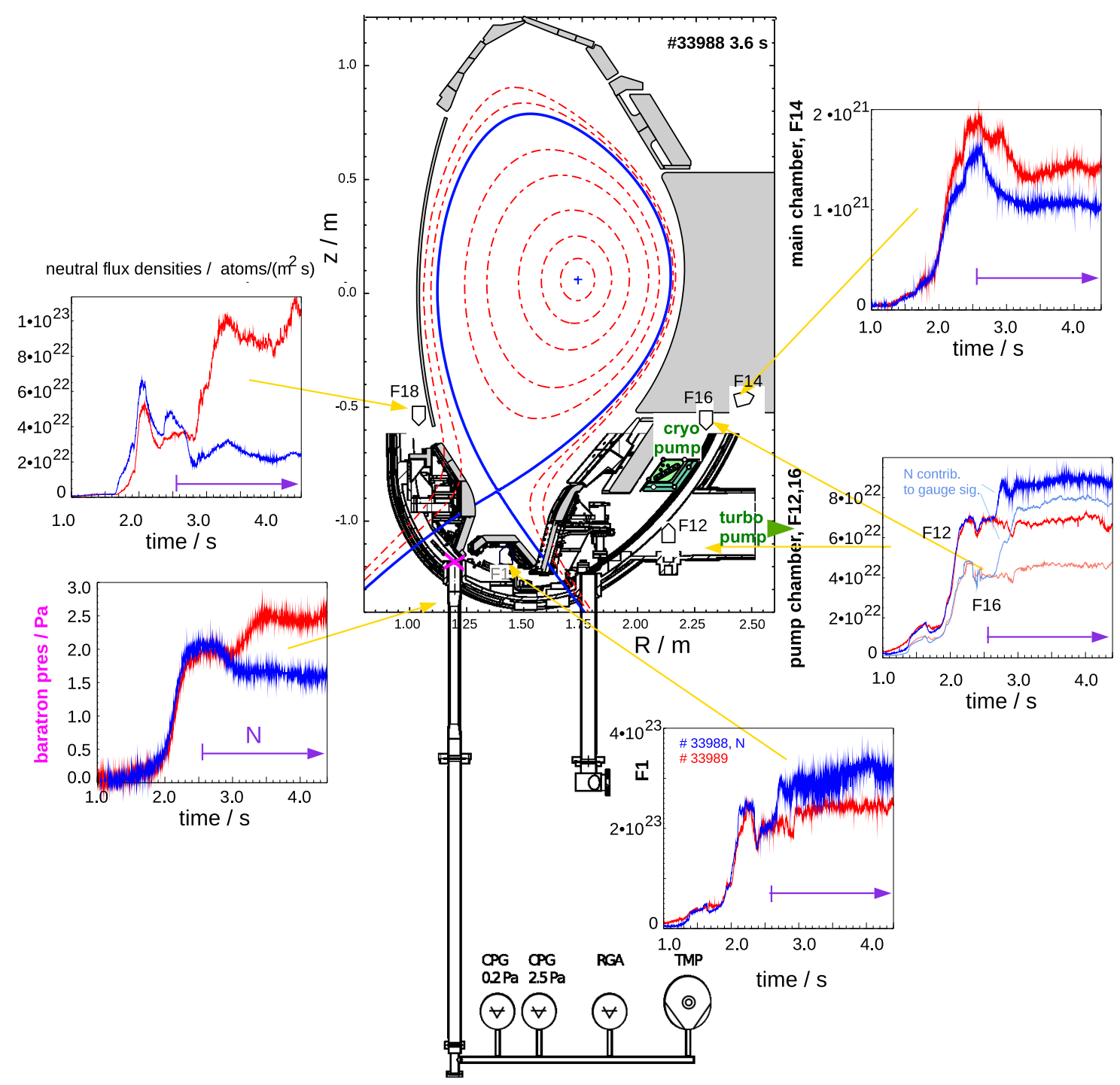

Figure 3. Experimental situation of pressure and flux density measurements in the lower divertor. The baratron measurement at the end of the HFS vertical port was calibrated to represent the pressure at the port entrance, marked by a magenta cross. The cryo pump (pumping speed $S \approx 100 \mathrm{~m}^{3} / \mathrm{s}$ ) and the turbo pumps $\left(S \approx 10 \mathrm{~m}^{3} / \mathrm{s}\right.$ ) are attached to the pumping chamber on the rhs of the outer divertor. The pumping chamber has a neutral conduction of about $25 \mathrm{~m}^{3} / \mathrm{s}$ to the sub roof baffle volume, thus a pressure drop by a factor $\approx 4$ is observed between sub roof baffle volume and pump chamber. Neutral fluxes are shown for the discharge pair introduced in figure 8. The ionisation gauges also measure nitrogen (sensitivity for $N$ pressure about a factor 2.4 higher compared to D), which explains the rise in the divertor gauge signals after start of the $N$ puff.

Good agreement is observed, albeit a trend towards higher values derived from Thomson scattering compared to the Eich prediction for higher plasma currents is visible. Different parameter variations have been tested to identify a physics parameter responsible for the moderate deviation from the Eich scaling at high $\mathrm{I}_{p}$. As shown in figure $2 \mathrm{~b}, \lambda_{q, \text { Spitzer }} / \lambda_{q, \text { Eich }}$ shows no significant trend with nitrogen content or degree of detachment (color coded). We conclude that the most probable cause of the deviation is 
the influence of ELMs on the measured temperature decay length. Such an influence is expected to be larger at higher $\mathrm{I}_{p}$, where the inter-ELM decay length is shorter. It should be noted that a corresponding deviation was not observed in the analysis shown in [19], where a more regular ELM behaviour allowed to leave out ELM-affected time points. The discharges shown here have acceptable or good H-mode confinement with $\mathrm{H}_{98} \geq$ 0.8 thanks to $\mathrm{N}$ seeding and high heating power. A broadening of $\lambda_{q, \text { Spitzer }}$ presented for a few discharges in [19] is connected to strong energy confinement degradation during detachment, with $\mathrm{H}_{98} \approx 0.5$. Obviously, the processes responsible for the pedestal degradation also increase $\lambda_{q}$.

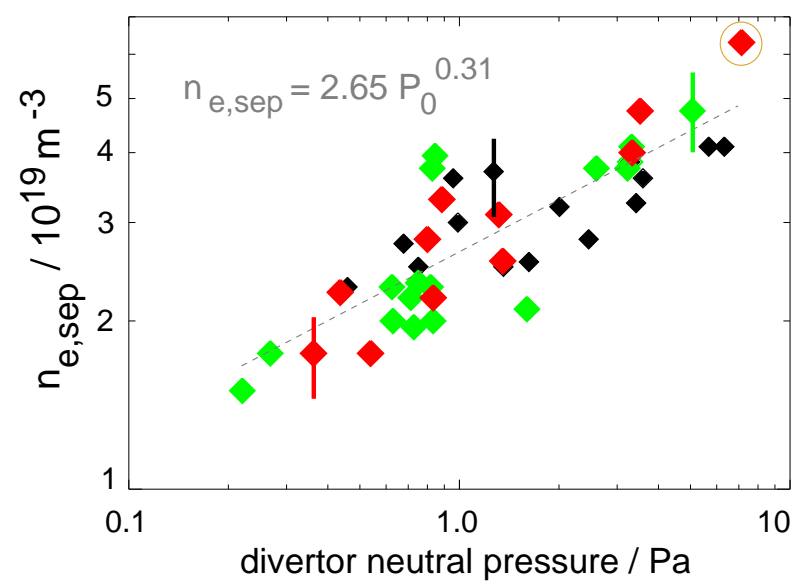

Figure 4. Dependence of the upstream separatrix density on divertor pressure, measured by a baratron below the roof baffle region. Coefficient of determination, $R^{2}$ $=0.7$, normalised root mean square deviation nrmsd $=17 \%$.

Various regression tests of the upstream separatrix density determined from Thomson scattering versus experimental parameters revealed the neutral divertor pressure, $\mathrm{p}_{0}$, as leading parameter. The pressure is measured by a baratron connected by a pipe below the high field side divertor, as shown in figure 3 , which also displays a plasma equilibrium and a simplified CAD view of the lower divertor. Time traces of pressure and neutral fluxes are also shown for a discharge being discussed below in section 4 . The baratron measurement at the end of a weakly pumped port far away from the lower divertor region, albeit being slower compared to ionization gauges, has several advantages. The measured pressure can safely be related to thermal neutral molecules of room temperature, therefore both pressure and flux density are known. The measured baratron pressure has been calibrated to represent the pressure at the entrance of the port on the high field side of the inner divertor, marked by a magenta cross in figure 3. The baratron pressure measurement remains correct also in the presence of nitrogen, while ionization gauges are disturbed by a higher sensitivity to $\mathrm{N}$ fluxes compared to $\mathrm{D}$ fluxes and thus do not allow a precise flux density measurement if a significant nitrogen concentration is present. Generally, the baratron measurement agrees with different ionisation gauges below the roof baffle in terms of atom flux densities better than a factor of 2 over a broad parameter range. This suggests that the net mass flows in this 
region are relatively small. The moderate deviation of the baratron during seeding is explained by its weak, but non-negligible neutral conductance to the region above the inner divertor, where the HFSHD produces strong pressure variations (see gauge F18).

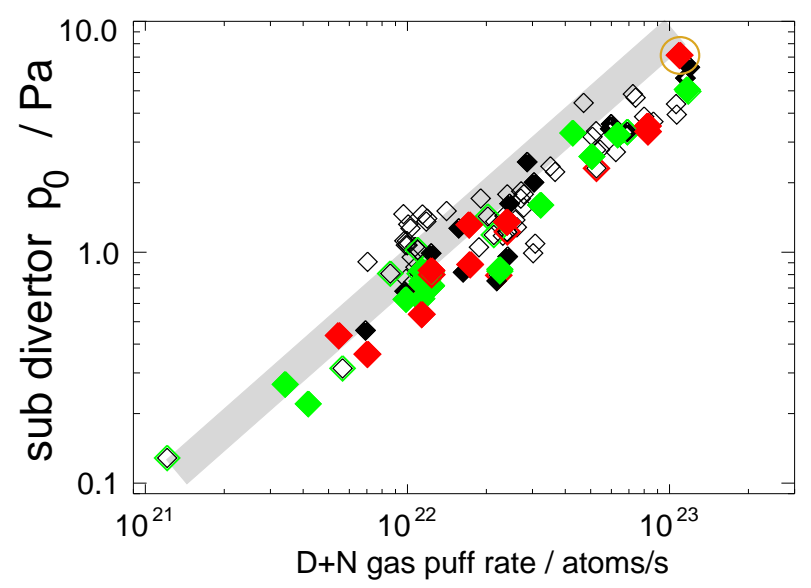

Figure 5. Dependence of the divertor pressure measured by a baratron below the roof baffle region and the sum of $N$ an $D$ gas puff rates. The grey line shows the expected pressure for a constant effective pumping speed of 20-30 $\mathrm{m}^{3} / \mathrm{s}$, which is the expected range with full cryopump active. For a few data points with 1/3 cryo pump active, the gas puff rates have been divided by the estimated effective pumping speed fraction, 0.5, for the shot with cryo off (marked by circle) by 0.3, respectively. Measurement uncertainties of pressure and gas fluxes are of the order of the symbol sizes. NBI fuelling has been neglected.

The neutral pressure below the roof baffle, $\mathrm{p}_{0}$, can be regarded as an engineering parameter since it is largely set by the gas puff level and the applied pumping speed. The roof baffle region comprises the connection to the pumping chamber by a toroidally revolving aperture with a neutral conductance of about $25 \mathrm{~m}^{3} / \mathrm{s}$, causing a pressure drop towards the pump chamber by about a factor 4 . A close correlation of the separatrix density with the neutral pressure $\mathrm{p}_{0}$ is found, as shown in figure 4 , the scaling obtained is

$$
n_{e, s e p}=2.65 p_{0}^{0.31}\left(10^{19} \mathrm{~m}^{-3}, P a\right)
$$

Despite some scattering, no systematic trends with other parameters were found, suggesting a close physics coupling of divertor neutral pressure and upstream separatrix density. The experimental trend of higher neutral pressure at higher current is produced by operational conditions (like, e.g., the necessity to puff gas to avoid tungsten accumulation at higher plasma currents). To first order, the same $\mathrm{n}_{e, \text { sep }}$ is obtained for the same $\mathrm{p}_{0}$ at high and low plasma currents.

In an earlier study, the average SOL density (line-averaged density over the first $6 \mathrm{~cm}$ outside the separatrix in the outer midplane) from Li beam measurements had been compared to the neutral flux density below the divertor roof baffle [1]. A relation $n_{e, S O L} \propto \Gamma_{0}^{0.55} q_{95}^{0.68}$ had been observed, not far from the present result except the $\mathrm{q}_{95}$ dependence. A possible explanation for this deviation may be the fact that the scrape-off 
layer density is effected/elevated by filaments leading to density shoulder formation.

In a simple picture of tokamak gas balance, in equilibrium the divertor neutral pressure is proportional to the gas puff rate, with the effective pumping speed as constant of proportionality. Figure 5 compares the measured neutral pressure with the gas puff rate. In reality, the simple relation is modified by uptake or release of gas by the walls [22] and a weak pressure dependence of the pumping speed. In addition, the pressure measured by the baratron is effected by neutrals produced in the vicinity of the high field side high density region, see section 4 .
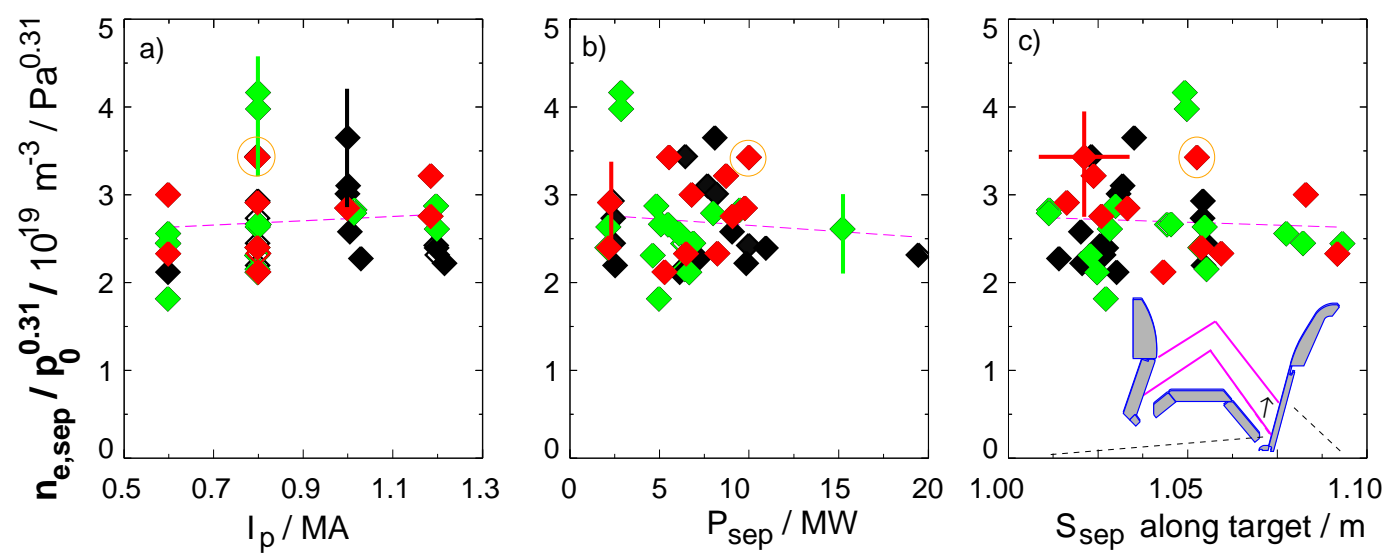

Figure 6. Separatrix density from Thomson scattering normalized by its neutral pressure dependence versus a) plasma current, $R^{2}=0.017$ b) separatrix power, $R^{2}$ $=0.01 \mathrm{c})$ position of the outer strike point along the target, $R^{2}=0.003$. The dashed lines are offset-linear fits, showing the weak dependence of the normalized $n_{\text {sep }}$ on $I_{p}$ and $P_{\text {sep }}$. nrmsd $=19 \%$ for a), b), c).

Figure 6 shows the separatrix density normalized by its neutral pressure dependence versus the plasma current, the non-radiated power crossing the separatrix and the strike point location at the target. The very weak dependence of the normalized separatrix density on $\mathrm{I}_{p}$ and $\mathrm{P}_{\text {sep }}$ suggests that the rise of $\mathrm{n}_{e, s e p}$ with $\mathrm{I}_{p}$ shown in figure 1 is caused by the increased gas puff / recycling level. There is also no significant dependence on the strike point location.

Since $\mathrm{n}_{e, s e p}$ was found to at most weakly depend on plasma current, the lineaveraged / pedestal density is investigated. Figure 7 shows the difference of line-averaged and separatrix density versus plasma current. An about linear rise is obtained, which is attributed to the confinement improvement / reduced particle transport with rising $\mathrm{I}_{p}$. Variations of the difference for a fixed $\mathrm{I}_{p}$ are at least partly related to different triangularities $\delta$ and an increase of the line-averaged during pronounced detachment [9].

\section{Expectations from simple analytical considerations}

In the following, the observed experimental trend between the upstream separatrix density $\mathrm{n}_{\text {mid }}$ and the neutral divertor pressure, $\mathrm{p}_{0}$ is derived using simple 2-point 


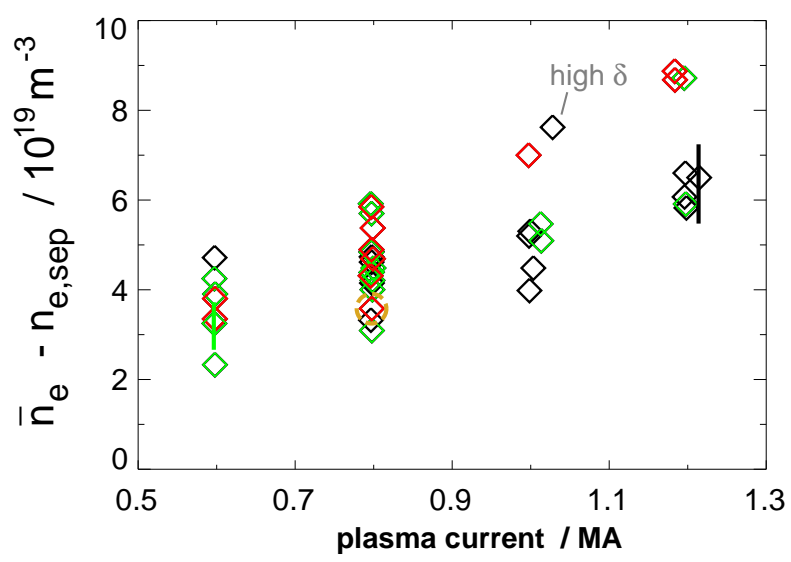

Figure 7. Difference of line-averaged and separatrix density versus plasma current.

model considerations [23], assuming dominant electron conduction and $\mathrm{T}_{e}=\mathrm{T}_{i}[\mathrm{eV}]$. The present treatment differs from recent work [16] regarding the use of 'enginering parameters', namely the neutral flux rather than the divertor $\mathrm{T}_{e}$.

Key element is the coupling of the plasma parameters in the divertor and midplane by pressure balance and heat conduction. Momentum losses and divertor radiation are considered via simple multiplier / loss factors $\mathrm{f}_{\text {mom }}$ and $\mathrm{f}_{r a d}$ between outer midplane and sheath in front of the target:

$$
f_{\text {mom }}=2 n_{\text {div }} T_{\text {div }} /\left(n_{\text {mid }} T_{\text {mid }}\right), \quad f_{\text {rad }}=1-b q_{\|, d i v} / q_{\|, m i d}
$$

A Mach=1 flow towards the target at the sheath is assumed, resulting in a reduction of the static pressure by a factor 2 . We have also introduced a divertor heat flux broadening factor $b=\lambda_{\text {int }} / \lambda_{q} \approx 1+1.64 S / \lambda_{q}$ with the exponential decay $\lambda_{q}$ and the Gaussian broadening $S$ [18] [24]. The midplane temperature is approximated assuming Spitzer electron conductivity along the connection length $\mathrm{L}$ and the divertor heat flux is given by the sheath boundary condition:

$$
\begin{aligned}
& e T_{\text {mid }}=e\left(\frac{7}{2 \kappa}\right)^{2 / 7}\left(L q_{\|, \text {mid }}\right)^{2 / 7} \\
& q_{\|, \text {div }}=\gamma\left(2 / m_{D}\right)^{0.5} n_{\text {div }}\left(e T_{\text {div }}\right)^{3 / 2}, \kappa=2380 \frac{W}{\mathrm{meV}^{7 / 2}}
\end{aligned}
$$

$\gamma$ is the sheath energy transmission factor which is typically between 7 and 8 , but can be as low as 4.6 for a tungsten surface when about $50 \%$ of the ion energy is reflected [25]. We abstain from using a flux limiter [15] for the electron parallel conductivity to keep the model simple and perspicuous. The omission of flux limiters leads to a moderate over-estimation of the parallel electron heat conductivity, which compensates in trend with the omission of ion parallel heat conduction, which starts to play a role if $\mathrm{T}_{i, m i d} \geq$ $2 \mathrm{~T}_{e, \text { mid }}$.

We want to establish a connection to the neutral pressure. For this we make the assumption that the neutral flux density, $\Gamma_{0}$, measured in the sub-divertor equals the ion flux density perpendicular to the target. This assumption applies to the 1D model 
geometry used here (flux densities correspond to total fluxes for given target surface area, particles are conserved), but has to be discussed for realistic conditions, see below.

$$
\Gamma_{0}=\sin (\alpha) q_{\|, d i v} /\left(\gamma e T_{d i v}\right)=\left(1-f_{\text {rad }}\right) \sin (\alpha) q_{\|, m i d} /\left(b \gamma e T_{\text {div }}\right)
$$

$\alpha$ is the impact angle of the field line at the outer target, a typical value is $2.5^{\circ}$. The radiative losses, which include in this simple treatment the power loss due to charge exchange and ionisation, are described by $\mathrm{f}_{\text {rad }}$. Finally, by combination of eqns 1-3, we obtain for the upstream density

$$
n_{\text {mid }}=\frac{2}{f_{\text {mom }}}\left(1-f_{\text {rad }}\right)^{1 / 2} \frac{1}{e}\left(\frac{2 \kappa}{7 L}\right)^{2 / 7}\left(m_{D} / 2\right)^{0.5}(b \gamma \sin (\alpha))^{-1 / 2} q_{\|, \text {mid }}^{3 / 14} \Gamma_{0}^{1 / 2}(6)
$$

Eq. 6 quite well reconciles the experimental finding for $n_{\text {mid }}$ shown in figure 4 that the separatrix density depends mainly on the neutral flux and at most weakly on plasma current and power. Eq. 6 predicts a weak $\mathrm{I}_{p}$ dependence expected via $q_{\|, \text {mid }} \propto P_{\text {sep }} / \lambda_{q}$, $\lambda_{q} \propto 1 / B_{p} \propto 1 / I_{p}$. The $\mathrm{f}_{\text {mom }}$ and $\mathrm{f}_{\text {rad }}$ dependencies partly cancel, since an increase in $\mathrm{f}_{\text {rad }}$ usually causes a reduction in $\mathrm{f}_{\text {mom }}$ [3]. A detailed discussion of the physics of the momentum losses in front of the target for pure plasma conditions can be found in [26]. The equation 6 predicts a dependence of the upstream separatrix density on the divertor heat flux broadening factor $b$. This factor is quite unknown for conditions of significant power and momentum losses where the Eich fit [18] can no longer be used to describe the power flux profile shape.

In the derivation of Eq. 6, the assumption was made that ion and neutral flux densities are equal in the strike point region. This cannot be expected for a realistic divertor geometry. Scarabosio et al. investigated the poloidal distribution of neutral fluxes in the AUG divertor for Ohmic an L-mode conditions [27]. For rising outer divertor density, the neutral flux density ratio beween the sub roof-baffle region and a location at the outer target decreases and becomes equal when the outer divertor detaches. This has been explained by the fact that the outer divertor plasma becomes transparent to neutrals at low $\mathrm{T}_{e}$. The interpretation of the absolute measurement value of the outer divertor gauge appeared difficult due to a factor 2-3 shortfall compared to the ion fluxes [27]. The gauge failed for the 2017 AUG campaign, so a neutral flux measurement close to the outer strike point was not available.

The poloidal distribution of the neutral fluxes in the sub-divertor volume was found to be quite uniform in the present study, when the gauge flux is converted to a pressure as measured by the baratron using the relation $\Gamma_{0}=\mathrm{p}_{0} 1.510^{23}$ atoms $\mathrm{m}^{-2} \mathrm{~s}^{-1} / \mathrm{Pa}$. A molecular flux at $\mathrm{T}=300 \mathrm{~K}$ at the baratron location is assumed. The baratron measurement is moderately affected by very strong variation of the neutral source in the high field side $\mathrm{X}$-point region related to the HFSHD, which reacts strongly to $\mathrm{N}$ puffing [20].

An explanation for the difference in the exponent for the neutral flux dependence between model eq. 6 and experiments (0.5 vs. 0.31 ) may be found in the variation of the neutral opacity at the outer strike point region. A quantitative assessment of this effect would require a 2D modelling with, e.g., the SOLPS code. Apart from the deviation in 
flux dependence, the analytical model well captures the observed insensitivity of $\mathrm{n}_{e, s e p}$ versus plasma current and power flux.

\section{Effects of seeding on neutral gas and fueling}

The effect of nitrogen seeding on the energy confinement has been at least partly attributed to a reduction of the separatrix density, which results in a favorable alignment of edge pressure and current profiles [2]. To shed further light on the relation of $\mathrm{N}$ seeding, neutral fluxes and $\mathrm{n}_{e, s e p}$, a well diagnosed discharge pair is investigated in more detail. Figure 8 compares traces of two discharges with and without nitrogen seeding using Tdiv feedback control [28]. Langmuir probe data along the outer target for both

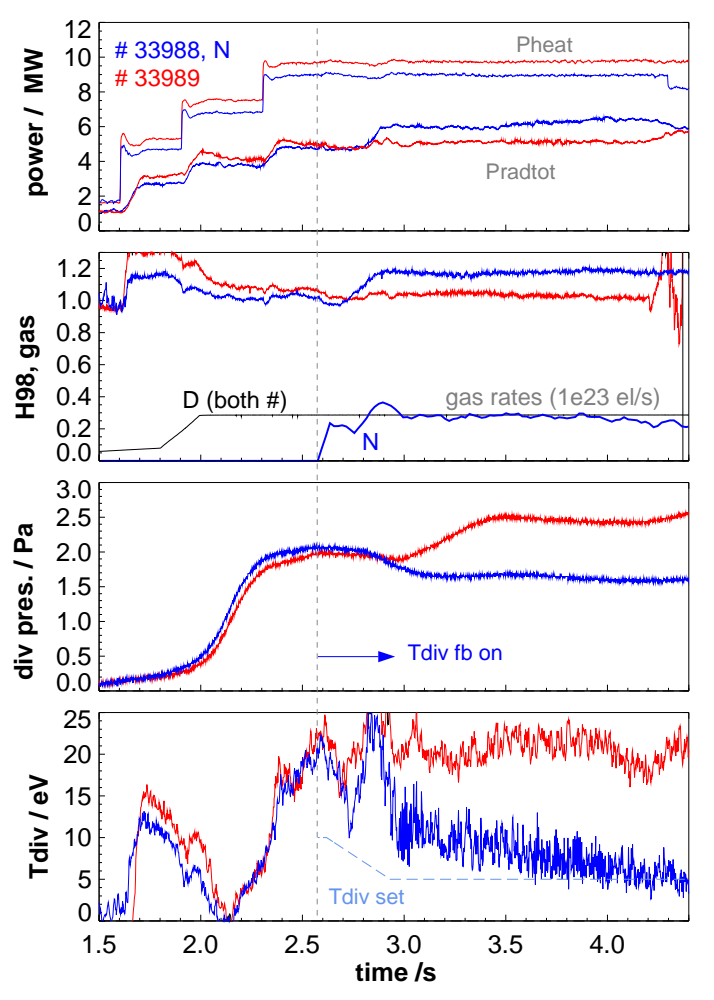

Figure 8. Comparisons of 2 discharges with and without $N$ seeding. Measured upstream separatrix densities from TS are 2.1 (with $N$ ) and $2.910^{19} \mathrm{~m}^{-3}$ (w/o N). $I_{p}=0.6 \mathrm{MA}$.

discharges are shown in figure 9. The seeding of nitrogen has an influence on the poloidal distribution of neutral fluxes, as shown in figure 3. The reduction of the neutral flux density below the inner heat shield (gauge F18) is attributed to a reduction of the high field side high density region [29] [20] [30]. This reduction also effects the baratron neutral pressure measurement below the high field side divertor region. In contrast, the ionisation gauge signals below the roof baffle (F1) and in the pump chamber (F12, 16) rise due to the contribution of nitrogen. It has to be noted here that the sensitivity of the pressure gauges is a factor $\approx 2.4$ higher for $\mathrm{N}$ compared to $\mathrm{D}$, resulting a significant 


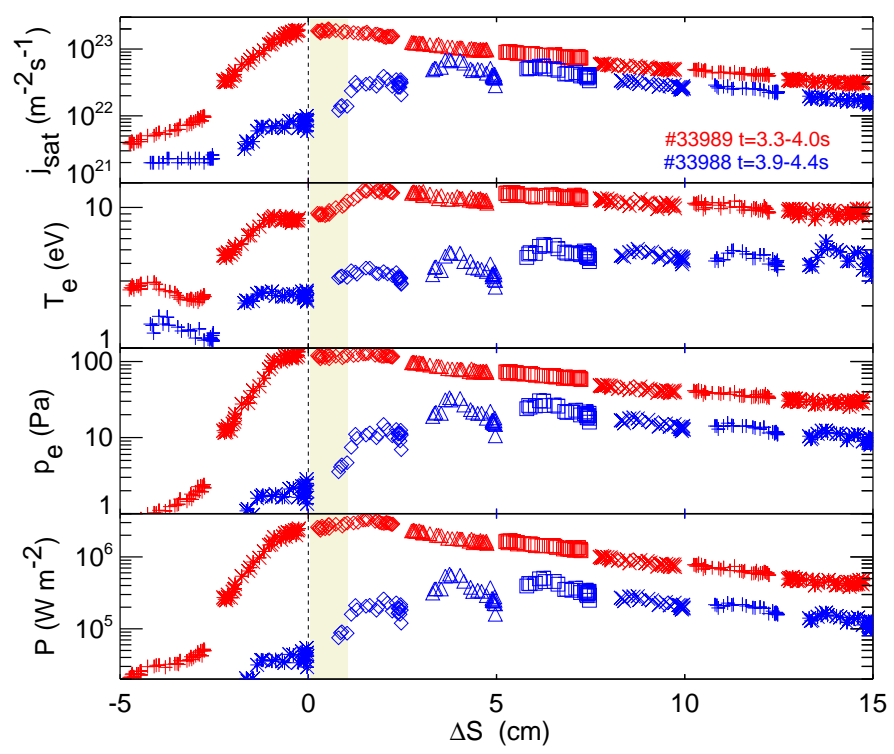

Figure 9. Langmuir triple probe data for the discharge pair introduced in figure 8. Data are median filtered over $20 \mathrm{~ms}$ to eliminate small excursions due to the small ELMs. The shaded bar indicates the first power e-folding length $\lambda_{q}$.

contribution of $\mathrm{N}$ to the measured flux density during strong $\mathrm{N}$ seeding. The neutral flux in the outer main chamber (gauge F14 in figure 3) is reduced during the N puff compared to the unpuffed case, which may be explained by the reduced separatrix density. Since the D puff is kept constant, particle balance suggests an about constant contribution of D to the sub divertor pressure signals with and without $N$ puffing. During $N$ seeding, the separatrix density decreases despite a constant D puff. This is attributed to the reduced fueling effect of the high field side high density [30]. The fact that this contribution is caught by using the neutral pressure at the location of the baratron in the scaling obtained may be coincidence. Currently, it is not clear to which extent the HFSHD contributes to $\mathrm{n}_{e, s e p}$ via neutral and via diffusive ion fueling.

\section{Conclusions}

Measurements of the upstream separatrix density for $\mathrm{N}$ seeded and unseeded $\mathrm{H}$-modes reveal a strong correlation with the divertor neutral pressure, $\mathrm{n}_{e, s e p} \propto p_{0, d i v}^{0.31}$ for the closed divertor condition in ASDEX Upgrade. The dependence on other experimental parameters appears relatively weak. The observed trend for increasing $\mathrm{n}_{e, s e p}$ with plasma current is mainly caused by operational conditions, which tend to use higher gas puff rates at higher $\mathrm{I}_{p}$. Under stationary conditions, the divertor pressure can be regarded as an engineering parameter being largely proportional to the gas puff rate. The measured relation of $\mathrm{n}_{e, s e p}$ and $p_{0, \text { div }}$ has been approximately reconciled by simple 2-point considerations. In the simple model, the upstream separatrix density is determined by the sheath boundary condition at the outer target, the assumption of Spitzer parallel electron heat conduction and pressure balance. The somewhat weaker experimental 
dependence of $\mathrm{n}_{e, \text { sep }}$ on the neutral pressure compared to 2-point modelling (exponent 0.31 vs. 0.5 ) may be explained by variations of divertor neutral opacity or other divertor plasma properties with the neutral pressure.

Since extrapolation from the target quite well captures midplane conditions, and also in line with [16], one may cautiously conclude that core fueling and transport effects are not very important for the value of the separatrix density. However, a moderate fueling contribution by the high field side high density seems likely, which results in a reduction of $\mathrm{n}_{e, s e p}$ with the neutral pressure at the HFS divertor region during nitrogen seeding [30]. The rise of the pedestal / line-averaged density with plasma current is related to transport effects between separatrix and pedestal top. The fact that the divertor neutral pressure acts as the dominant engineering parameter for the separatrix density, and also determines the outer divertor detachment threshold [10] can serve as a guideline for future optimization of tokamak performance. The separatrix density is limited by $\mathrm{H}$-mode confinement degradation and the $\mathrm{H}-\mathrm{L}$ transition [8]. With the relation obtained in this work, this limit can also be connected to a divertor neutral pressure limit. The neutral pressure on the other hand is also a leading parameter for the divertor power exhaust and achievement of detachment [9] and can thus be regarded as key engineering parameter in operational scenario optimization. Further work is required on the divertor heat flux broadening (factor b), which may also effect the relationship of neutral pressure and $\mathrm{n}_{e, \text { sep }}$. Maximizing the neutral pressure for given $\mathrm{n}_{e, \text { sep }}$ may be the path to optimize power exhaust in terms of achieving the highest possible detachment power for given seed impurity concentration. $2 \mathrm{D}$ modelling and possibly additional physics (turbulence, blobs) will have to be considered to understand present experiments and to gain a better predictive capability.

\section{Acknowledgements}

This work has been carried out within the framework of the EUROfusion Consortium and has received funding from the Euratom research and training programme 2014-2018 under grant agreement number 633053. The views and opinions expressed herein do not necessarily reflect those of the European Commission.

\section{References}

[1] SCHWEINZER, J. et al., Journal of Nuclear Materials 266-269 (1999) 934.

[2] DUNNE, M. G. et al., Plasma Physics and Controlled Fusion 59 (2017) 014017.

[3] LIPSCHULTZ, B. et al., Fusion Science and Technology 51 (2007) 369.

[4] REINKE, M., Nucl. Fusion (2017) 034004.

[5] GOLDSton, R. et al., Plasma Phys. Controlled Fusion (2017) 055015.

[6] PSHENOV, A. et al., Phys. Plasmas 24 (2017) 072508.

[7] BERNERT, M. et al., Plasma Physics and Controlled Fusion 57 (2015) 014038. 
[8] EICH, T. et al., Correlation of the tokamak H-mode density limit with ballooning stability at the separatrix, accepted for publication in Nuclear Fusion (2018).

[9] KALLENBACH, A. et al., Nuclear Fusion 55 (2015) 053026.

[10] KALlENBACH, A. et al., Plasma Physics and Controlled Fusion 58 (2016) 045013.

[11] SAMM, U. et al., Journal of Nuclear Materials 176-177 (1990) 273.

[12] KALLENBACH, A. et al., Journal of Nuclear Materials 415 (2011) S19.

[13] REIMOLD, F. et al., Journal of Nuclear Materials 463 (2015) 128.

[14] NEUHAUSER, J. et al., Plasma Physics and Controlled Fusion 44 (2002) 855.

[15] STANGEBY, P. C. et al., Nucl. Fusion 55 (2015) 093014.

[16] LEONARD, A. et al., Nucl. Fusion 57 (2017) 086033.

[17] SUN, H. J. et al., Plasma Physics and Controlled Fusion 59 (2017) 105010.

[18] EICH, T. et al., Phys. Rev. Lett. 107 (2011) 215001.

[19] SUN, H. J. et al., Plasma Physics and Controlled Fusion 57 (2015) 125011.

[20] POTZEL, S. et al., Journal of Nuclear Materials 463 (2015) 541.

[21] EICH, T. et al., Nuclear Fusion 53 (2013) 093031.

[22] ROHDE, V. et al., Plasma Physics and Controlled Fusion 51 (2009) 124033 (8pp).

[23] STANGeBY, P., The Plasma Boundary of Magnetic Fusion Devices, Institute of Physics Publishing, Bristol and Philadelphia, 2000.

[24] MAKOWSKI, M. et al., Phys. Plasmas 19 (2012) 056122.

[25] BRIDA, D. et al., Nucl. Fusion 57 (2017) 116006.

[26] KOTOV, V. and REITER, D., Plasma Phys. Controlled Fusion (2009) 115002.

[27] SCARABOSIO, A. et al., Journal of Nuclear Materials 390-391 (2009) 494.

[28] KALlENBACH, A. et al., Plasma Physics and Controlled Fusion 52 (2010) 055002.

[29] POTZEL, S. et al., Nuclear Fusion 54 (2014) 013001.

[30] REIMOLD, F. et al., Nuclear Materials and Energy 12 (2017) 193. 